(RESEARCH ARTICLE)

\title{
Experimental characterization and modelling of microfluidic radial diffusers for passive self-regulating valves
}

\author{
Chappel Eric ${ }^{1,}{ }^{*}$, Musard Hugo ${ }^{1,2}$ and Dumont-Fillon Dimitry ${ }^{1}$ \\ ${ }^{1}$ Debiotech SA, 28 avenue de Sévelin, Lausanne 1004, Switzerland. \\ ${ }^{2}$ LMSI4, EPFL, Swiss Federal Institute of Technology, Lausanne 1015, Switzerland.
}

Publication history: Received on 14 October 2019; revised on 22 November 2019; accepted on 30 November 2019

Article DOI: https://doi.org/10.30574/gjeta.2019.1.1.0002

\begin{abstract}
A microfluidic radial diffuser typically comprises a hole in a membrane facing a concentric pillar separated by a small gap where the fluid is forced to flow radially between the membrane and the pillar. Such diffusers are notably used to make passive flow rectification valves for drug delivery devices, wherein several holes are machined into a flexible membrane progressively deflecting against pillars as the pressure increases on top of it. The fluidic modelling of such a diffuser is made difficult by the presence of a transition region between the hole and the diffuser itself. An experimental investigation has been conducted using SOI wafers to form membranes having only one central hole and Pyrex wafers for the substrate and pillars. Both wafers are anodically bonded together after alignment. A simple fluidic model accounting for the specific geometry of the diffuser is proposed and compared to experimental results. A good match is obtained, for Reynolds number in the range 0.5 to 35 , using the analytical formula of a radial diffuser in the laminar regime with an effective inner radius $40 \%$ smaller than the actual one, in order to correctly simulate the flow constriction at the entrance of the diffuser.
\end{abstract}

Keywords: Medical devices; Hydrodynamics; Laminar Flow; Microsystems; Micro Electro Mechanical Systems (MEMS); Micromanufacturing

\section{Introduction}

Passive flow rectification or flow control microvalves are microfluidic chips that can be used for cerebrospinal fluid management or drug delivery [1-2]. A typical application is an implantable pump for pain management. Such devices usually comprise a reservoir pressurized with liquefied gas and a flow restrictor. It is well known that these devices are sensitive to temperature variations. Flow rate variations up to $+/-13 \%$ per ${ }^{\circ} \mathrm{C}$ in body temperature change are reported [3]. Those systems will also deliver a different drug amount in environmental conditions of high pressures (scuba diving) or after a long exposition to high altitudes (up to $+35 \%$ of over-delivery) [3]. Therefore, patients are advised to avoid these conditions. However, replacing the flow restrictor by a flow control valve makes the device insensitive to pressure variations. In addition, to limit under or overdoses, the implantable pump with a flow control valve offers the possibility to lower the reservoir pressure so as to limit risks during drug refilling process. Specific flow control valves may also comprise a passive compensation of the temperature effect on viscosity [4]. Microfluidic autoregulation obtained using the non-Newtonian rheological properties of polymeric solutions was reported by Groisman et al. [5, 6]. For the passive flow rectification of Newtonian fluids, different designs were reported, including 3D microfluidic devices made of stacks of PDMS layers [7-9], devices comprising spiral-shaped fluidic channels [10-12] or MEMS devices with micro-valves associated in parallel $[1,2,4,13]$.

Passive flow control can be achieved using a flexible silicon membrane having several through holes, each one being coupled with a concentric facing pillar etched in the substrate. The gap between the membrane and the top surface of the pillar depends on the reservoir pressure in direct communication with the top surface of the membrane. Increasing

\footnotetext{
${ }^{*}$ Corresponding author

E-mail address: e.chappel@debiotech.com
} 
this pressure induces a change of the fluidic restriction hereafter referred to as "radial diffuser" or simply "diffuser" (see Fig. 1). This diffuser is significantly different from the typical conical diffuser valves described in the literature, which are mainly used for micropump applications [14-21]. The design of the device, including the determination of the number of hole/pillar pairs and their radial location along the membrane, may be achieved using a genetic algorithm as it is a powerful tool to get the configuration exhibiting the lowest sensitivity to machining tolerances [13]. A first fluidic model has been provided for devices with gap values (up to $30 \mu \mathrm{m}$ ) in the same order of magnitude as the membrane thickness itself. Flow control valves dedicated to the infusion of viscous drugs at high pressure lead to the use of very small gaps (typically $10 \mu \mathrm{m}$ ) and thick membranes (up to $130 \mu \mathrm{m}$ ). Such a specific geometry requires an improved fluidic model that better takes into account the abrupt change of flow direction as well as the very large flow constriction at the diffuser entrance. Microfluidic radial diffusers comprising a single hole at the center of the membrane and a single pillar have been produced and characterized. After a brief description of the process flow and the different designs investigated, the fluidic characterization of the samples is provided along with the new fluidic modelling of the radial diffuser.

Table 1 Notations

\begin{tabular}{cl}
\hline Symbol & \\
\hline$\eta$ & dynamic viscosity \\
$R_{h}$ & fluid volumetric mass \\
$L_{h}$ & hole radius \\
$R_{p}$ & hole depth \\
$\mathrm{h}$ & pillar radius \\
$\mathrm{d}$ & Distance between the pillar and the membrane \\
$P_{\text {in }}-P_{\text {out }}$ & valve off-centering \\
$Q_{i}$ & Pressure gradient \\
$w_{c}$ & flow rate via the fluidic pathway $i$ \\
$h_{c}$ & channel width \\
$L_{c}$ & channel height \\
$R_{f \text { out }}$ & channel length \\
$\kappa$ & fluidic resistance of the outlet \\
& correction parameter
\end{tabular}

\section{Fluidic Model}

\subsection{Radial Diffuser}

A schematic cross-section of the valve is shown in Fig. 1. The different notations in the text are given in Table I.

Flow control valve dedicated to high-pressure applications usually exhibits a small gap $h$ :

$h \ll R_{h}, R_{p}, L_{h} \ldots$

This specific geometry shall be analyzed further in order to model the different head losses. 


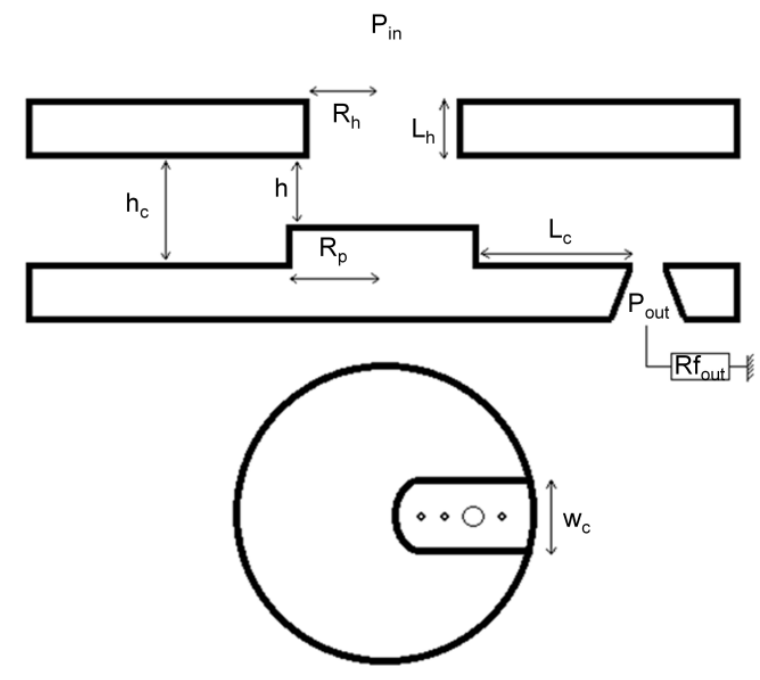

Figure 1 Schematic cross-section of a valve (top) and top view of the substrate having three pillars and one outlet hole inside a channel of width $w_{c}$ (bottom)

The difference in pressure $\Delta P=P_{\text {in }}-P_{\text {out }}$ between the inlet and the outlet of the valve is written as a function of $Q_{i}$ as follows:

$\Delta P=\alpha_{i} Q_{i}^{2}+\beta_{i} Q_{i}$

where $i$ indicates one fluidic pathway, $\beta_{i}=\sum_{i} R f_{i}$ is the sum of the fluidic resistances of the fluidic pathways $i$, and $\alpha_{\mathrm{i}}$ is a function of the surfaces of each singularity. We estimate numerically the function $\alpha_{i} Q_{i}$ for each value of $\Delta \mathrm{P}$ :

$\alpha_{i} Q_{i}=-\frac{\beta_{i}}{2}+\sqrt{\alpha_{i} \Delta P+\frac{\beta_{i}^{2}}{4}}$

The total flow rate is, therefore:

$Q=\sum_{i} Q_{i}$

We consider now a flow regulator that comprises several hole/pillar pairs.

\subsection{Singular Head Losses}

Fig. 2 (a) shows a schematic illustration of a radial diffuser, where the reservoir connected to the hole is at the pressure $\mathrm{P}$, the cone represents the flow constriction before the diffuser itself and finally, the channel communicates with the outlet. The location of the different sources of singular head losses are noted, respectively: $A$ for the contraction from the reservoir to the hole, $B$ for the progressive contraction between the hole and the diffuser, $C$ for the bend at the diffuser entrance, $D$ for the radial expansion inside the diffuser and $E$ for the vertical expansion from the diffuser to the channel. We define the cone opening angle $\gamma$ as follows (see also Fig. 3):

$\gamma=2 \arctan \left(\frac{4 \kappa R_{h i}}{L_{h}}\right)$

Before entering into the diffuser, the flow experiences a bend with an angle equal to $\frac{\pi-\gamma}{2}$. According to [22], in the bend, the expression of the singular coefficient takes the form:

$\sin ^{2}\left(\frac{\pi-\gamma}{4}\right)+2 \sin ^{4}\left(\frac{\pi-\gamma}{4}\right) \cdot(6)$ 


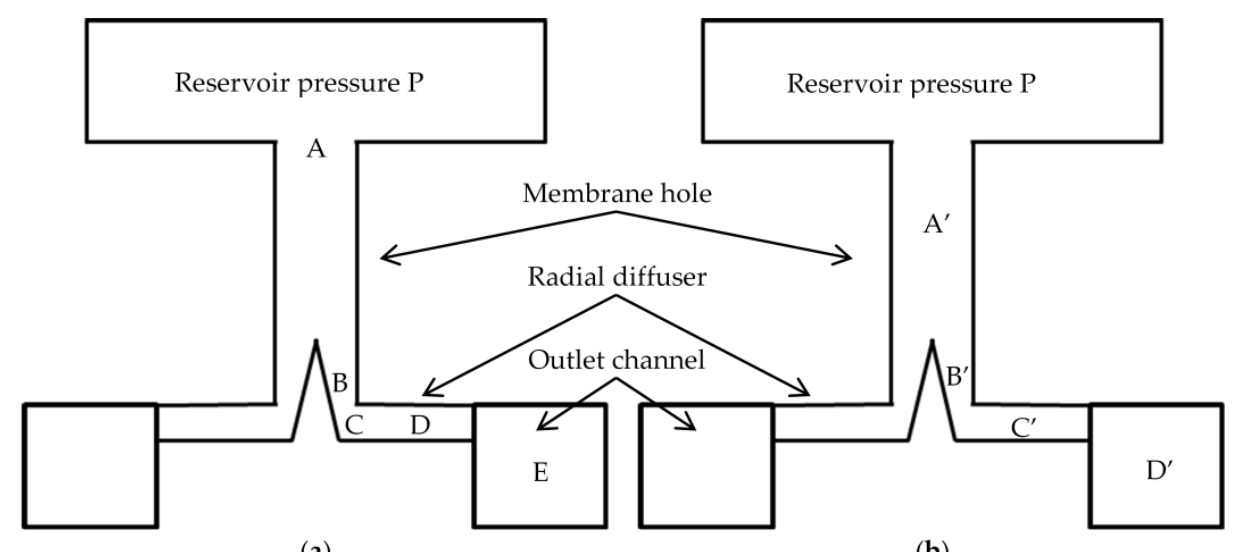

(a)

(b)

Figure 2 Schematic representations of the singular (a) and linear (b) head losses of a radial microfluidic diffuser. Each letter corresponds to a specific loss described in the text

For positive gradients of pressure, i.e. when the fluid flows through the hole, the diffuser, the fluidic channel and finally the outlet hole, the parameters $\alpha_{i}$ defined in (2) take the form (see [1] for the different expressions used):

$\alpha_{i}=\frac{\rho}{2 S^{2}}(A+B+C+D+E)=\frac{\rho}{2}\left(\frac{1}{\pi^{2} R_{h i}^{4}}+\frac{0.4}{\pi^{2}}\left(\frac{1}{\kappa^{2} R_{h i}^{2}-\left(\kappa R_{h i}-h_{i}\right)^{2}}-\frac{1}{\kappa^{2} R_{h i}^{2}}\right)^{2}\left(1-\frac{h_{i}}{\kappa R_{h i}}\right)^{2} \sin (\gamma)+\frac{\sin ^{2}\left(\frac{\pi-\gamma}{4}\right)+2 \sin ^{4}\left(\frac{\pi-\gamma}{4}\right)}{4 \pi^{2} \kappa^{2} R_{h i}^{2} h_{i}^{2}}+\frac{1}{4 \pi^{2} h_{i}^{2}}\left(\frac{1}{R_{p i}}-\right.\right.$

$\left.\left.\frac{1}{\kappa R_{h i}}\right)^{2}+\frac{\left(1-\frac{h_{i}}{h_{c}}\right)^{2}}{4 \pi^{2} R_{p i}^{2} h_{i}^{2}}\right) \cdot(7)$

where the terms $A$ to $E$ are the contributions of the fluidic elements shown in Fig. 2 (a).

The parameter $\kappa$ has been introduced to better fit the experimental data when the gap is much smaller than the hole radius and the channel height, even at zero pressure. In that case, the parameter $\alpha_{i}$ can be simplified as follows:

$\alpha_{i}=\frac{\rho}{2}\left(\frac{\sin ^{2}\left(\frac{\pi-\gamma}{4}\right)+2 \sin ^{4}\left(\frac{\pi-\gamma}{4}\right)+0.4 \sin (\gamma)}{4 \pi^{2} \kappa^{2} R_{h i}^{2} h_{i}^{2}}+\frac{1}{4 \pi^{2} h_{i}^{2}}\left(\frac{1}{R_{p i}}-\frac{1}{\kappa R_{h i}}\right)^{2}+\frac{1}{4 \pi^{2} R_{p i}^{2} h_{i}^{2}}\right)$.

\subsection{Linear Head Losses}

The linear losses due to the fluid viscosity take place in four different locations as depicted in Fig. 2 (b): $A^{\prime}$ is the flow through the hole, $B^{\prime}$ is the flow constriction between the hole and the radial diffuser entrance (conical shape), $C^{\prime}$ is the flow through the radial diffuser, and $D^{\prime}$ is the flow in the outlet channel.

The parameters $\beta_{i}$ defined in (2) take the form (see [1] for the different expressions used):

$$
\begin{aligned}
& \beta_{i}=A^{\prime}+B^{\prime}+C^{\prime}+D^{\prime}=\frac{8 \eta\left(\frac{3}{4} L_{h}+h_{i}\right)}{\pi R_{h i}^{4}} \\
& +\int_{0}^{\frac{L_{h}}{4}} \frac{8 \eta}{\pi}\left(1 /\left(\kappa^{4} R_{h i}^{4}-b(x)^{4}-\left(\frac{\left(\kappa^{2} R_{h i}^{2}-b(x)^{2}\right)^{2}}{\ln \left(\frac{\kappa R_{h i}}{b(x)}\right)}\right)\right)\right) d x+\frac{6 \eta}{\pi h_{i}^{3}} \ln \left(\frac{R_{p i}}{\kappa R_{h i}}\right)+\frac{12 \eta L_{c i}}{w_{c i} h_{c i}^{3}} .
\end{aligned}
$$

where the terms $A^{\prime}$ to $D^{\prime}$ are the contributions of the fluidic elements shown in Fig. 2 (b). Fig. 3 shows a schematic representation of the flow through the cone, which is an idealized representation of the transition between the hole and the diffuser entrance.

The parameter $b(x)$ used in the expression of the term $B^{\prime}$ takes the form: 
$b(x)=\left\{\begin{array}{cc}\frac{4\left(\kappa R_{h i}-h_{i}\right)}{L_{h}} & \text { if } \kappa R_{h i}-h_{i} \geq 0 \\ 0 & \text { otherwise }\end{array}\right.$

We also consider a fluidic resistance at the outlet $R f_{\text {out }}$ (due to the test setup or the connection to the patient for an infusion device). In that case, for a given pressure gradient $\Delta P$, we estimate the flow rate $Q$ as shown previously.

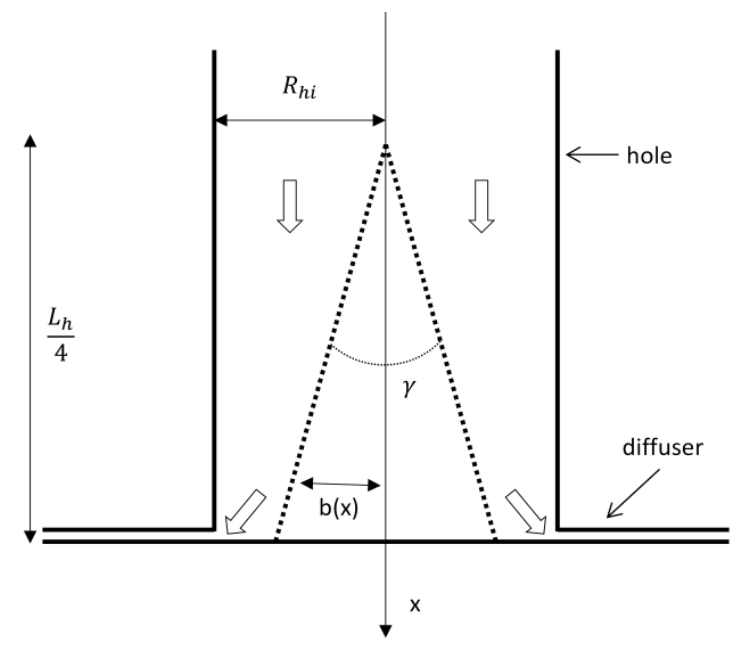

Figure 3 Schematic cross-section of the idealized flow transition between the hole and the diffuser entrance. The flow direction is indicated by arrows. The dashed line is an idealized representation of the transition between the hole and the diffuser entrance.

The additional pressure drop $\Delta P_{\text {out }}$ due to $R f_{\text {out }}$ is:

$\Delta P_{\text {out }}=R f_{\text {out }} Q$

And the effective gradient of pressure necessary to get the flow rate $Q$ is, therefore:

$\Delta P_{\text {eff }}=\Delta P+\Delta P_{\text {out }}$

\section{Design and microfabrication}

\subsection{Design Description of the Prototypes}

Specific designs of microfluidic radial diffusers have been made to better understand the fluidic behavior of such valves and to improve the fluidic modelling by adding a new parameter $\kappa$ intended to be determined using experimental data. This parameter is a means to better simulate the flow constriction at the entrance of the diffuser, in particular, if the gap is small compared to other dimensions.

To prevent any error due to the estimation of the gap as the pressure increases, it has been decided to produce test devices having only one central hole/pillar pair. Up to the contact between the membrane and the substrate, the analytical formula giving the deflection as a function of the pressure $p$ is valid as long as the deflection does not exceed $0.5 t$, where $t$ is the membrane thickness [23]:

$Z_{\max }=\frac{3}{16} \frac{p R^{4}\left(1-v^{2}\right)}{E t^{3}}$

Where $E$ is Young's modulus, $v$ is Poisson's ratio, and $R$ is the membrane radius. The pillar height is fixed at $50 \mu \mathrm{m}$ for all devices. The average measured dimensions of the samples are provided in Table II. The gap is measured with a mechanical profiler (Bruker Dektak XT surface profiler). The membrane thickness is obtained from the certificate of conformity of the SOI wafers, while the other dimensions are measured using an optical microscope Nikon Eclipse NV150 (software Kappa Metreo). The ratio pillar radius/hole radius is in the range of 1.25 to 2.8. 
Table 2 Key Dimensions of the Devices D1 to D7

\begin{tabular}{llllll}
\hline $\begin{array}{l}\text { Device } \\
\#\end{array}$ & $\begin{array}{l}\text { Membrane } \\
\text { thickness } \\
(\boldsymbol{\mu m})\end{array}$ & $\begin{array}{l}\text { Membrane } \\
\text { radius } \\
(\boldsymbol{\mu m})\end{array}$ & $\begin{array}{l}\text { Gap } \\
(\boldsymbol{\mu m})\end{array}$ & $\begin{array}{l}\text { Hole } \\
\text { radius } \\
(\boldsymbol{\mu m})\end{array}$ & $\begin{array}{l}\text { Pillar } \\
\text { radius } \\
(\boldsymbol{\mu m})\end{array}$ \\
\hline D1 & 30 & 1879 & 10.4 & 48.4 & 138 \\
D2 & 30 & 1580 & 10.2 & 48.4 & 137 \\
D3 & 30 & 1428 & 10.3 & 48.3 & 137 \\
D4 & 30 & 1329 & 10.4 & 48.4 & 136 \\
D5 & 30 & 1257 & 10.3 & 48.3 & 137 \\
D6 & 129.5 & 2560 & 10.3 & 48.4 & 132 \\
D7 & 129.5 & 2560 & 10.8 & 61.5 & 77.5 \\
\hline
\end{tabular}

A schematic cross-section of a device D6 is provided in Fig. 4a. The chip dimensions in mm are $10 \times 10 \times 1(L \times W \times H)$. The membrane (top wafer) contains one single inlet hole while the substrate has two outlet holes (to improve the priming of the device). A zoom of the radial diffuser at rest (membrane hole and pillar) is shown in Fig. $4 \mathrm{~b}$.

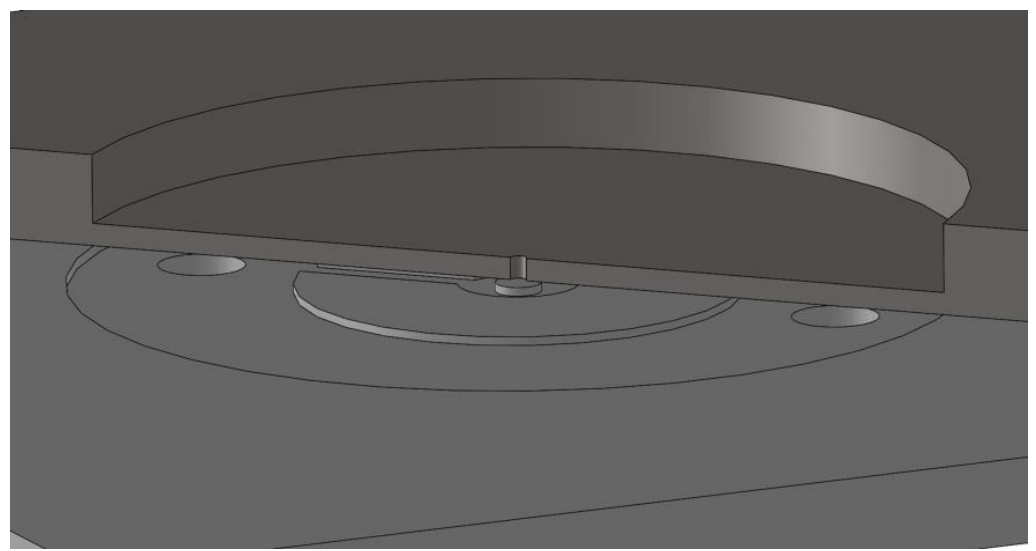

Figure 4a Schematic cross-section of the device D6 (membrane = top wafer, substrate = bottom wafer).

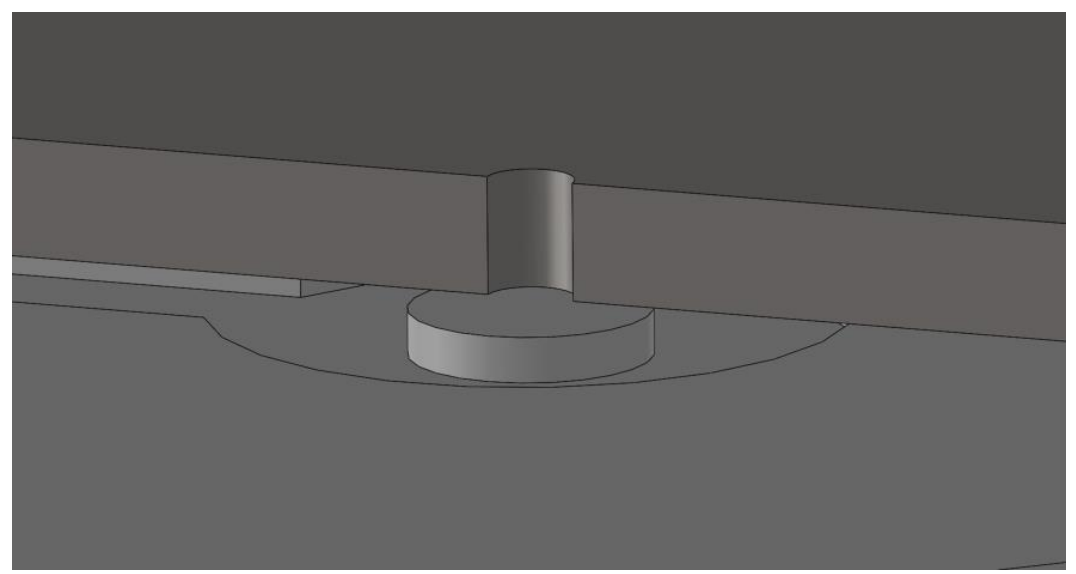

Figure 4b Zoom of the radial diffuser D6 


\subsection{Manufacturing Process Flow}

The holes and the membrane are made by dry etching (DRIE, Bosch process on Alcatel AMS200) of a 4" SOI wafers (front side and backside respectively). Two different SOI device layers have been considered: $30 \mu \mathrm{m}$ and $129.5 \mu \mathrm{m}$. The buried oxide is $2 \mu \mathrm{m}$ thick. Residues are removed with $\mathrm{O}_{2}$ plasma and the final silicon oxide wet etching is obtained using a BHF 7:1 solution. The gap and pillars are obtained through isotropic wet etching (HF $10 \%$ at room temperature) of a 4" glass wafer (Borofloat 33, thickness $500 \mu \mathrm{m}$ ). The metallic hard mask used for the pillar definition was opened by dry etching (ion beam Veeco Nexus IBE350). The outlet hole (500 $\mu \mathrm{m}$ in diameter) in the glass substrate is made by sandblasting (IcoFlex Sarl, Ecublens, Switzerland). The two wafers are finally aligned at $\pm 10 \mu \mathrm{m}$ and anodically bonded together (Süss BA6/SB6). Chip singulation is performed using an automatic dicing saw Disco DAD321.

\section{Fluidic characterization}

The devices have been tested with filtered water at $20^{\circ} \mathrm{C}$. The water tank is pressurized by a pressure controller Druck DPI520 connected to high-pressure $N_{2}$ bottle (200 bar). A sample holder similar to the one described in [2] has been used for the tests. The flow rate is estimated by infusing at constant pressure during 1 minute into a beaker placed onto a scale Sartorius AC210P. The pressure ranges are 0 to 400 mbar for the devices D1 to D5 and 0 to 2.5 bar for the devices D6 and D7.

Typical flow rate versus pressure profiles measured using the thin (30 $\mu \mathrm{m}$ - devices D1 to D5) and thick (129.5 $\mu \mathrm{m}-$ devices D6 and D7) membranes are provided in Figures 5 and 6 respectively. Considering the different devices, the maximum Reynolds number varies within the range 0.5 to 35 .

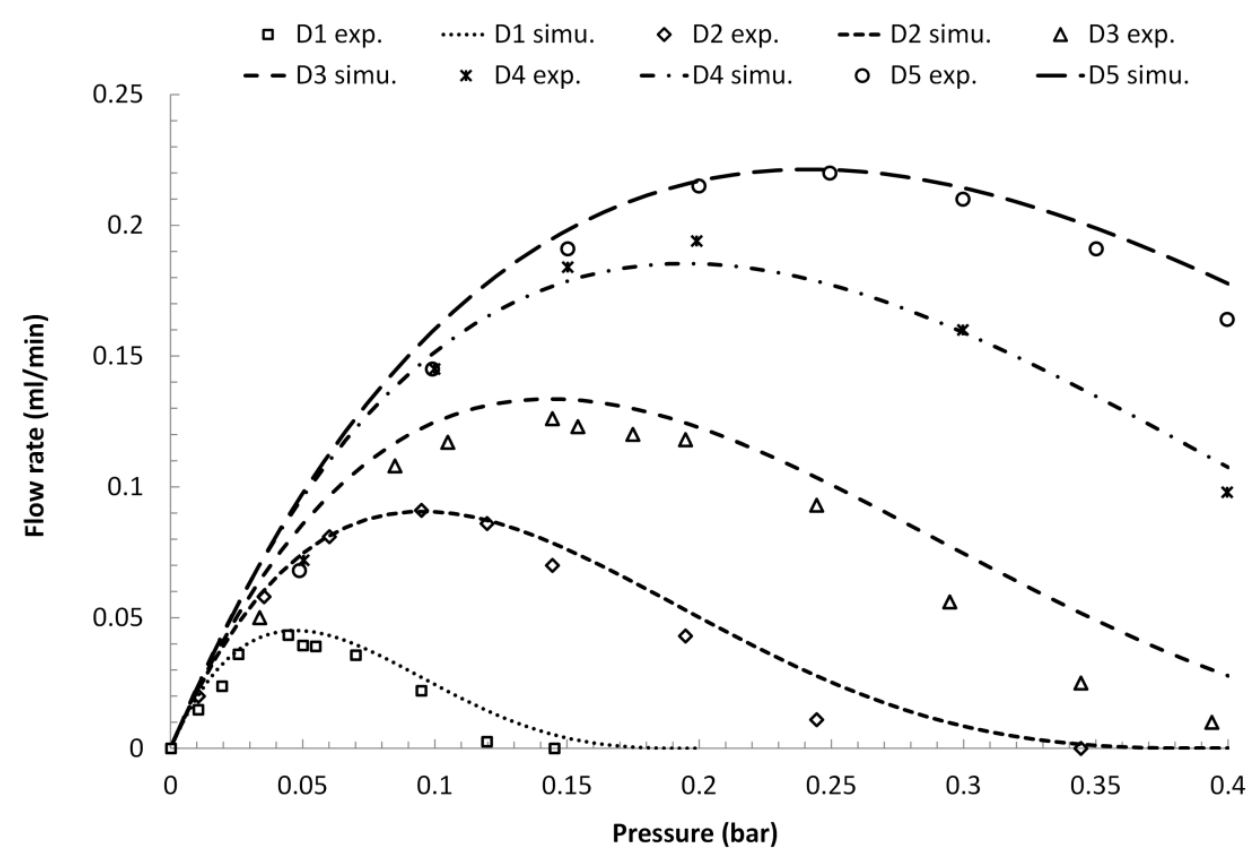

Figure 5 Flow rate versus pressure profiles of the devices D1 to D5 and comparison with numerical simulations using

$$
\kappa=0.6
$$




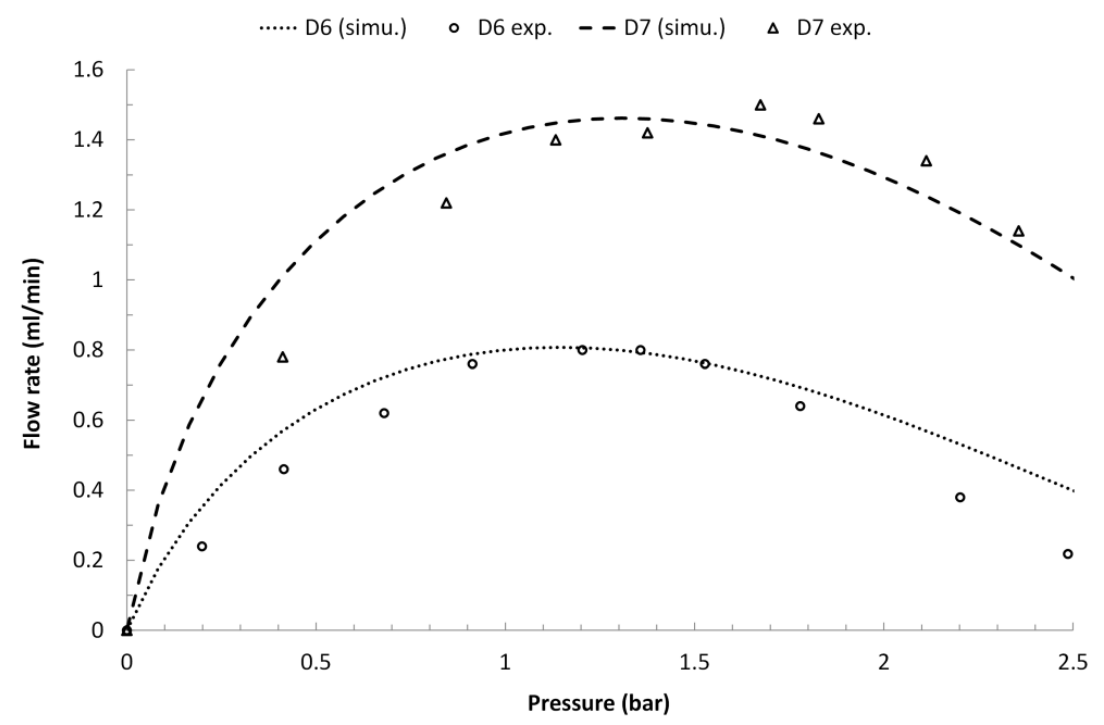

Figure 6 Flow rate versus pressure profiles of the devices D6 and D7 and comparison with numerical simulations using $\kappa=0.6$

\section{Results and discussion}

The $Q(\Delta P)$ profiles shown in Figures 5 and 6 exhibit an asymmetric dome shape. By contrast to other radial valves wherein the fluidic behavior at low pressure is driven by the constant fluidic resistance of the membrane holes [1], we observe here that there is no linear regime even at low pressure. All tested devices exhibit a small value of the initial gap between the membrane and the pillar (about $10 \mu \mathrm{m}$ ) and a large membrane hole diameter $(>96 \mu \mathrm{m}$ ). The fluidic resistance of the diffuser itself is therefore always dominant. Since the gap $h$ decreases linearly with the applied pressure gradient $\Delta P$ (before contact), the fluidic resistance of this diffuser will increase as $h$ (and therefore $P$ ) to the power -3 , preventing any linear regime in the fluidic characteristic of the devices.

Considering in a first and basic approximation that the flow resistance of the valve can be written:

$R_{f} \approx \frac{a}{h^{3}}(14)$

Where $a$ is a constant and $h$ is the gap equal to the difference between the gap at rest $h_{0}$ and the deflection of the center of the membrane defined in Eq. (13):

$h=h_{0}-z_{\max }=h_{0}-u \Delta P$

$u$ is a constant that depends on the membrane characteristics (see Eq. (13).

The flow rate in the valve can be approximated by a function having the following form:

$Q=\frac{\Delta P}{R_{f}} \approx \frac{\Delta P}{a}\left(h_{0}-u \Delta P\right)^{3}$

The devices D1 to D5 are similar except the membrane radius $R$, therefore the parameter $u$ can be written:

$u=c R^{4}$

Where $c$ is a constant.

By replacing Eq. (17) in Eq. (16) we obtain:

$Q \approx \frac{\Delta P}{a}\left(h_{0}-c R^{4} \Delta P\right)^{3}$ 
The derivative of this function with respect to the pressure gradient is:

$\frac{d Q}{d(\Delta P)} \approx \frac{\left(h_{0}-4 c R^{4} \Delta P\right)\left(h_{0}-c R^{4} \Delta P\right)^{2}}{a}$

According to this approximation, the maximum flow rate is observed at a pressure gradient $\Delta P\left(Q_{\max }\right)$ which corresponds to the first root of this derivative:

$\Delta P\left(Q_{\max }\right) \approx \frac{h_{0}}{4 c R^{4}}$

The second root of this derivative corresponds to the pressure $\Delta P\left(Q_{\text {vanish }}\right)$ at which the flowrate vanishes:

$\Delta P\left(Q_{\text {vanish }}\right) \approx \frac{h_{0}}{c R^{4}}$

Since both $\Delta P\left(Q_{\text {van }}\right)$ and $\Delta P\left(Q_{\max }\right)$ are proportional to the membrane radius $R$ at the power -4 , the larger the value of the membrane radius the smaller the value of the pressure at which the flow rate vanishes and the value of the pressure at the top of the dome as observed experimentally.

The introduction of $\Delta P\left(Q_{\max }\right)$ (see Eq. 20) in the equation (18) leads to a value of $Q_{\max }$ that is also proportional to $1 / R^{4}$ :

$Q_{\max } \approx \frac{27 h_{0}{ }^{4}}{256 a c R^{4}}$

The simplified model introduced at the beginning of this section is, therefore, useful to explain the trend of the experimental curves, including the fact that the bigger membranes have the smallest flow rates, in good agreement with the experimental observations (see Figure 5).

However, in order to fit the experimental data, the whole expressions of the singular and linear head losses shall be used. The average measured misalignment of $10 \mu \mathrm{m}$ between the pillars and the membrane holes has also been introduced into the model. The gap varies in principle between $h_{0}$ (at rest) and 0 (at high pressure, when the membrane is in contact with the pillar). Since we use a particle filter that exhibits a pore size of $0.2 \mu \mathrm{m}$, we fix the minimum gap to 0.2 instead of $0 \mu \mathrm{m}$, assuming that contamination could prevent the total closure of the valve.

As mentioned in the introduction, the original fluidic modelling (see [1]) is not adapted to a radial diffuser that exhibits a gap that is much smaller than all other dimensions of the devices. A parameter $\kappa$ has therefore been introduced into the model as shown in section II, to better account for the flow constriction at the diffuser entrance. The data analysis has been carried out focusing on the central part of the curves, for pressure values centered around $\Delta P\left(Q_{\max }\right)$, since the measurement errors are smaller at high flowrate. Moreover, a flow control valve usually includes several radial diffusers, and the behavior of the valve is mainly secured by the radial diffuser that is located near the outer edge of the membrane. The flow rate through this diffuser remains large at high pressure while all other radial diffusers located nearer to the center of the membrane are almost closed (these other radial diffusers are only used to fine-tune the flow profile at low pressure). Therefore the overall fluidic characteristic of the valve is driven by the high flow parts of the different radial diffusers (the diffusers located near the membrane centre at low pressure and the outer diffuser at high pressure). Considering this limitation, a good match between experiment and simulation (in Matlab) is obtained using $\kappa=0.6$. It should be noted that the use of a single parameter is not sufficient to perfectly fit the tail of the different curves at high pressure.

\subsection{Application to the characterization of the non-linear deformation of a membrane against a rigid substrate}

As mentioned in [23], the highly non-linear deflection of a clamped membrane against a rigid substrate has no simple analytical solution. FEM simulations can be carried out to determine, at different test pressures, the gap between the membrane and the substrate as a function of the radial position, but the experimental validation of the modelling is relatively complex, in particular at high pressure. To improve the reliability of the gap estimation as a function of the applied pressure, it is proposed to perform the fluidic characterization of chips that exhibit the same radial diffusers located at different radial positions.

We first assume that the correction due to the membrane tilt is small (membrane diameter 》 gap) [2]. 
Description of the "fluidic" method to derive the non-linear deformation of the membrane:

A fluidic model for the valve is established by measuring the flow rate versus pressure characteristic of the chip with the centered diffuser: the evolution of the fluidic resistance of the valve as a function of the gap $R_{f}($ gap) is derived since the deflection of the center of a clamped membrane is well known [23].

The fluidic resistance of the same (i.e. same geometry) but off-centered diffuser is measured: for this given radial position of the diffuser the function $R_{f}$ (pressure) is derived.

With each value of the fluidic resistance is associated a value of the gap using the interpolation of the function $R_{f}(g a p)$ : the function gap (pressure) is therefore derived for a given value of the nominal gap and for a given membrane (material, diameter, and thickness).

This method was applied to the diffuser design D6 (see Table II for the diffuser design). A similar diffuser had previously been used in a flow control valve (see reference [2], design A, with a nominal flow rate of $0.5 \mathrm{~mL} / \mathrm{min}$ at $12 \mathrm{cP}$ ). There is however a small discrepancy regarding the pillar diameter $(-15 \%)$ due to over-etch during the processing of the samples D6.

Samples were made according to the process flow described in $\S 3$. We consider a diffuser design D6 located at a distance of $2.09 \mathrm{~mm}$ from the membrane center. The diffuser was characterized by the test setup described in reference [2] up to 18 bar. The value of the "fluidic" gap versus the fluidic resistance was derived using the data provided in Figure 6. Finally, the interpolated value of the "fluidic" gap between the membrane and the pillar at the radial position $2.09 \mathrm{~mm}$ was derived using this "fluidic" probe (see Figure 7). Such an indirect estimation of the membrane deflection ("fluidic probe") could be a simple alternative to the development of a specific and complex characterization setup at high pressure. This method is indeed a bypass to the direct measurement of the real gap: the function gap (pressure) can be introduced into the fluidic model described in section 2. By definition, the model is able to reproduce the experimental data obtained during the fluidic test of this specific diffuser, but errors due to interpolation and to the approximation made in the fluidic model are expected if the diffuser dimensions and/or radial position are modified. If the nominal value of the gap is small compared to all other dimensions, its value is usually the critical-to-quality parameter that shall be controlled in fabrication. Moreover, to determine the ideal value of the diffuser gap, it is recommended to investigate experimentally the effect of small variations of this parameter onto the fluidic characteristic of the microfluidic chips having such radial diffusers (the initial nominal gap value being derived from simulations). This method is of course only relevant in case of highly non-linear deformation of the membrane.

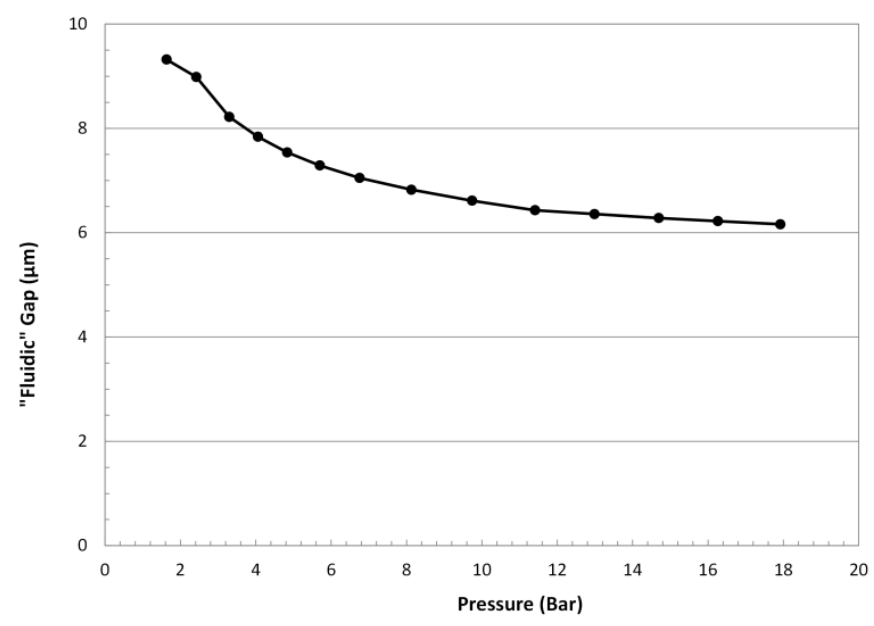

Figure 7 Interpolated value of the "fluidic" gap between the membrane and the pillar at the radial position 2.09 mm as a function of the applied pressure for a diffuser design D6 (see Table II). 


\section{Conclusion}

The numerical modelling of a microfluidic radial diffuser formed of a gap, much smaller than other critical dimensions, between a membrane and a pillar has been investigated. We observe, based on the analysis of the experimental data provided in Figs. 5 and 6, that the linear losses at the interconnection between the hole and the diffuser are simulated considering an increase of the constriction length of the diffuser. A single parameter $\kappa$ has been used to adjust the model accordingly to the experimental data. The expressions of the singular losses account for the specific geometry of the problem (especially the bend at the diffuser's entry and the diffuser itself). A match between modelling and experiments better than 15\% for a Reynolds number varying from 0.5 to 35 and a ratio pillar radius/hole radius in the range of 1.25 to 2.8 has been obtained. At very high pressure, when the valve is almost closed, additional corrections to the model are necessary to simulate the radial diffuser which becomes really sensitive to particulate contamination and surface roughness. The association of several microfluidic diffusers inside a single membrane will allow to obtain a constant flow rate over a predefined range of pressure. This analytical model of the radial diffuser is useful to design a microfluidic chip without complex 3D FEM simulations and metrology tools.

\section{Compliance with ethical standards}

\section{Acknowledgments}

The authors wish to thank Harald van Lintel and Philippe Renaud for fruitful discussions regarding the manufacturing process flow and the design (LMIS4, Swiss Federal Institute of Technology EPFL, Lausanne 1015, Switzerland), as well as Stephan Gamper and the staff of Center of MicroNanoTechnology at EPFL for support.

\section{Disclosure of conflict of interest}

The authors declare no conflict of interest.

\section{References}

[1] Chappel E, Dumont-Fillon D and Mefti S. (2014). Passive flow regulators for drug delivery and hydrocephalus treatment. In: Proc. SPIE 8976, Microfluidics, BioMEMS, and Medical Microsystems XII, paper no. 89760S.

[2] Cornaggia L, Conti L, Hannebelle M, Gamper S, Dumont-Fillon D, van Lintel H, Renaud P and Chappel E. (2017). Passive flow control valve for protein delivery. Cogent Engineering, 4(1), 1413923.

[3] Ethans KD, Schryvers OI, Nance PW and Casey AR. (2005). Intrathecal drug therapy using the Codman Model 3000 Constant Flow Implantable Infusion Pumps: experience with 17 cases. Spinal Cord, 43, 214-218.

[4] Chappel E. (2017). Adjustable passive flow regulator. European Patent No. EP2943708B1.

[5] Groisman A, Enzelberger M and Quake SR. (2003). Microfluidic memory and control devices. Science 300(5621), 955-958.

[6] Groisman A and Quake AR. (2004). A Microfluidic Rectifier: Anisotropic Flow Resistance at Low Reynolds Numbers. Physical Review Letters, 92, 094501.

[7] Karlalov EP, Walker C, Taylor CR, Anderson WF and Sherer A. (2006). Microfluidic vias enable nested bioarrays and autoregulatory devices in Newtonian fluids. Proceedings of the National Academy of Sciences USA, 103(33), 12280-12284.

[8] Zhang X, Xiang N, Tang W, Huang D, Wang X, Yi H and Ni Z. (2015). A passive flow regulator with low threshold pressure for high-throughput inertial isolation of microbeads. Lab on a Chip, 15, 3473-3480.

[9] Doh I and Cho YH. (2009). Passive flow-rate regulator using pressure dependent autonomous deflection of parallel membrane valves. Lab on a Chip, 9(14), 2070-2075.

[10] Amacker C, Leungki YS, Pasquita V, Madore C, Haller M and Renaud P. (1998). Passive Micro-Flow Regulator for Drug Delivery System. In: Proc. of Eurosensors XII, 591-594.

[11] Cousseau P, Hirschi R, Frehner B, Gamper S and Maillefer D. (2001). Improved micro-flow regulator for drug delivery systems. In: Proc. 14th IEEE International Conference on Micro Electro Mechanical Systems (MEMS) MEMS 2001, 527-531. 
[12] Chappel E. (2016). Design and characterization of a passive flow control valve dedicated to the hydrocephalus treatment. Cogent Engineering, 3(1), 1247612.

[13] Dumont-Fillon D, Hannebelle M, van Lintel H and Chappel E. (2016). Design of a Passive Flow Regulator Using a Genetic Algorithm. Procedia Engineering, 168, 1016-1019.

[14] Stemme E and Stemme G. (1993). A valveless diffuser/nozzle fluid pump. Sensors Actuators A, 39, $159-167$.

[15] Gerlach T. (1998). Microdiffusers as dynamic passive valves for micropump applications. Sensors Actuators A. 69, 181-191.

[16] Olsson A, Stemme G and Stemme E. (1996). Diffuser-element design investigation for valve-less pumps. Sensors Actuators A, 57, 137-143.

[17] Olsson A, Stemme G and Stemme E. (2000). Numerical and experimental studies of flatwalled diffuser elements for valve-less micropumps. Sensors Actuators A, 84, 165-175.

[18] Heschel M, Müllenborn M and Bouwstra S. (1997). Fabrication and characterization of truly 3-D diffuser/nozzle microstructures in silicon. J Microelectromech Syst, 6, 41-47.

[19] Jiang XN, Zhou ZY, Huang XY, Li Y, Yang Y and Liu CY. (1998). Micronozzle/diffuser flow and its application in micro valveless pumps. Sensors Actuators A, 70, 81-87.

[20] Rosa S and Pinho FT. (2006). Pressure drop coefficient of laminar Newtonian flow in axisymmetric diffusers. Int J Heat Fluid Flow, 27, 319-328.

[21] Wang YC, Hsu JC, Kuo PC and Lee YC. (2009). Loss characteristics and flow rectification property of diffuser valves for micropump applications. Int J Heat Mass Transfer, 52, 328-336.

[22] Ouziaux R and Perrier J. (1998). Mécanique des fluides appliquée, 3rd edition. Dunod, Paris, France, 141.

[23] Timoshenko SP and Woinowsky-Krieger S. (1959). Theory and plates and shells, Second edition. McGraw-Hill, New-York, USA, 55.

\section{How to cite this article}

Chappel E, Musard H and Dumont-FD (2019). Experimental characterization and modelling of microfluidic radial diffusers for passive self-regulating valves. Global Journal of Engineering and Technology Advances, 1(1), 10-21. 